\title{
MulTiNet: Multimodal Neural Networks for Glaucoma Based on Transfer Learning
}

\author{
Yi Li ( $\square$ yilineu@163.com ) \\ Northeastern University \\ YuJie Han \\ Northeastern University \\ XueSi Zhao \\ Northeastern University \\ ZiHan Li \\ Northeastern University \\ ZhiFen Guo \\ Northeastern University
}

\section{Research Article}

Keywords: Multi-Modal Classification Network, DualSY Database, Transfer Learning, Heidelberg OCT, Three-dimensional Fundus Photos

Posted Date: December 23rd, 2021

DOI: https://doi.org/10.21203/rs.3.rs-1164184/v1

License: (c) (i) This work is licensed under a Creative Commons Attribution 4.0 International License.

Read Full License 


\title{
RESEARCH
}

\section{MulTiNet: Multimodal Neural Networks for Glaucoma Based on Transfer Learning}

\author{
Yi Li ${ }^{*}{ }^{*}$, YuJie Han ${ }^{2}$, XueSi Zhao ${ }^{3}, Z_{i H a n} \mathrm{Li}^{4}$ and ZhiFen Guo ${ }^{5}$
}

\footnotetext{
${ }^{*}$ Correspondence: yilineu@163.com

${ }^{1}$ Department of Information

Science and Engineering,

Northeastern University, Shen

Yang, China

Full list of author information is

available at the end of the article
}

\begin{abstract}
Background: Being one of the most serious causes of irreversible blindness, glaucoma has many subtypes and complex symptoms. In clinic, doctors usually need to use a variety of medical images for diagnosis. Optical Coherence Tomography $(O C T)$, Visual Field $(V F)$, Fundus Photosexams $(F P)$ and Ultrasonic BioMicroscope $(U B M)$ are widely-used and complementary techniques for diagnosing glaucoma.
\end{abstract}

Methods: At present, the field of intelligent diagnosis of glaucoma is limited by two major problems. One is the small number of data sets, and the other is the low diagnostic accuracy of Single-Modal Modal.

In order to solve the above two problems, we have done the following work. First,we construct DualSY glaucoma multimodal data set. The four most important subtypes of glaucoma are discussed in this article which are Primary Open Angle Glaucoma $(P O A G)$, Primary Angle Closure Glaucoma $(P A C G)$, Primary Angle Closure Suspect $(P A C S)$ and Primary Angle Closure $(P A C)$. Each patient in the DualSY data set contains more than five medical images, as shown in the figure 4.And Dual $S Y$ are labeled with image-level multi-labels.

Second, We propose a new Multi-Modal classification network for glaucoma, which is a multiclass classification model with various medical images of glaucoma patients and text information as input. The network structure consists of three main branches to deal with patient metadata, domain-based glaucoma features and medical images. Transfer learning method is introduced into this paper due to the small number of medical image data sets. The flowchart is shown in Figure 5.

Result: Our method on glaucoma diagnosis outperforms state-of-the-art methods. A promising average result of overall accuracy $(A C C)$ of $94.7 \%$ is obtained. Our data set outperformed most data sets in glaucoma diagnosis with an accuracy of $87.8 \%$.

Conclusions: The results suggest that medical images such as Heidelberg OCT and three-dimensional fundus photos used in this paper can better express the high-level information of glaucoma and our modal greatly improve the accuracy of glaucoma diagnosis. At the same time, this data set has great potential, and we continue to study this data.

Keywords: Multi-Modal Classification Network; DualSY Database; Transfer Learning; Heidelberg OCT; Three-dimensional Fundus Photos 


\section{Introduction}

Glaucoma is an irreversible neuro-degenerative eye disease that is considered one of the main reasons of visual disability in the world [1] . According to the statistics of the World Health Organization(WHO), there are as many as 78 million glaucoma patients in the world in 2020 [2].

The recent advancements made in deep learning have been applied to different medical fields in order to detect (at an earlier stage) or predict certain anomalies [3-5]. In the ophthalmological domain, digital fundus images analysis using deep learning methods have started to receive a lot of attention. Among the main ophthalmological anomalies, glaucoma is one the most severe conditions, potentially leading to irreversible damage of the human vision as it affects the optic nerve fibers, responsible for conducting the sensory information related to the visual sense. Being an asymptomatic illness, the majority of the affected patients do not perceive conspicuous signs and they usually discover the symptoms when it is too late [6]. Simultaneously, early detection and treatment are important to prevent vision loss.

How to diagnose glaucoma as early as possible and improve the diagnostic accuracy has become an urgent problem to be solved in glaucoma diagnosis.

The main feature of glaucoma is loss of visual field, which is caused by increased intraocular pressure $(I O P)$ and/or loss of optic nerve blood flow [7]. However, IOP is found to be neither specific nor sensitive enough to be an effective glaucoma indicator since visual damage still be present with normal IOP. Glaucoma can also be diagnosed by measuring the Glaucoma angle, the thickness of optic nerve fiber layer and other characteristics. However, the diagnostic accuracy of glaucoma is low due to complex symptoms.

Previous studies [8] have shown that the accuracy of neural network based on color FP [9] and Heidelberg OCT [10] for early diagnosis of glaucoma is close to or better than that of ophthalmologists. At the same time, the algorithm can also predict the future disease changes such as the degree of visual field defect and the thickness of nerve fiber layer based on the baseline examination results. Based on high-quality multimodal image data and combined with neural network, it is expected to greatly improve the accuracy of diagnosis of glaucoma patients. The combination of artificial intelligence and medical imaging has brought breakthrough innovation to the clinical diagnosis and treatment of glaucoma.Therefore, it is of great practical significance to develop a multi-modal glaucoma diagnosis network system based on transfer learning.

Transfer learning mimics the human vision system by making use of sufficient amounts of prior knowledge in other related domains when executing new tasks in the given domain. In this paper, transfer learning is used, which can also quickly train an ideal effect when the number of medical image data sets is small.

At the same time, the published data set is limited to two categories of labels, which is not suitable for clinical application and has not been refined to glaucoma subtypes [11-18]. Therefore, we use the multi-modal and multi-label data set collected by the Fourth People's Hospital of Shenyang to study the diagnosis of glaucoma.

The main contributions of the article are summarized as follows: 


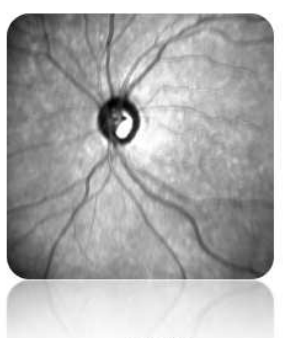

a. $O C T$

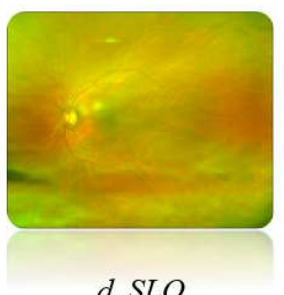

d. SLO

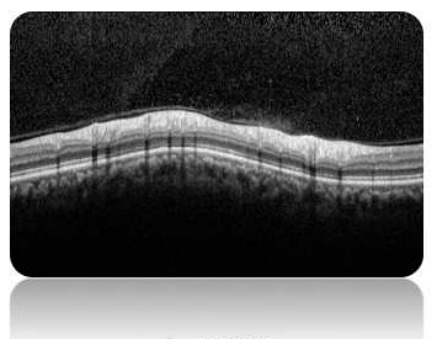

b. RNFL

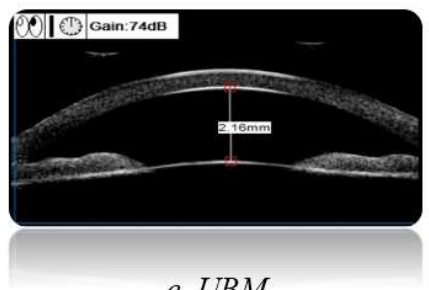

e. $U B M$

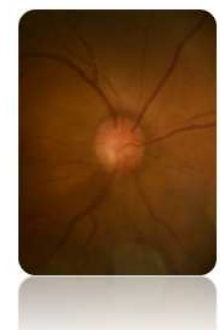

c. fungus photos

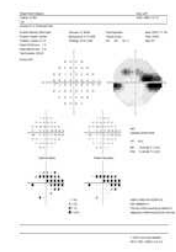

f. perimetry

Figure 1 Sample dataset.

a. Heidelberg Optical Coherence Tomography scanner.b.Picture of Retinal Nerve Fiber Layer.c.Three-dimensional Fundus Photo.d.Scanning Laser Ophthalmoscope.e.Ultra-Sonic Biomicroscopy.f.Perimetry.

- In this article,a brand-new glaucoma data set is constructed with five labels and multiple medical images,as shown in the figure 1 and figure 4 . As far as we know, this is the first multi-labels glaucoma data set, and the first dataset goes deep into the glaucoma subtype.

- At the same time,a multi-modal neural network is established to make multimodal accurate diagnosis of glaucoma. Three-branch structure is embedded in the network, and the text information and image information are fused at the end.

- We adopt a variety of strategies to improve the accuracy of our proposed model,including data augmentation, regularization, optimizer selection, evaluation index selection, learning rate adjustment and a loss function handling imbalanced data.

The richness of DualSY data set is unmatched by the known open source glaucoma data set. It not only provides researchers with richer medical images and glaucoma features, but also makes it possible to accurately diagnose glaucoma by using MultiModal Networks.Our multimodal intelligent glaucoma diagnosis network uses text and image information to diagnose glaucoma, which opens up a new avenue for glaucoma diagnosis. The results of experiments show that our method on glaucoma diagnosis outperforms state-of-the-art methods with the highest ACC. 
Table 1 List of all the public available databases with glaucoma.

\begin{tabular}{|c|c|c|c|c|}
\hline $\begin{array}{c}\text { Dataset } \\
\text { name }\end{array}$ & Paper & Quantity & Pixels & Remarks \\
\hline Drishti-GS & $\begin{array}{l}\text { Drishti-GS: Retinal } \\
\text { image dataset for optic } \\
\text { nerve head }(\mathrm{ONH}) \\
\text { segmentation }[11]\end{array}$ & 101 & $2896 \times 1944$ & $\begin{array}{c}\text { All images were taken } \\
\text { centered on OD with a } \\
\text { Field-Of-View(FOV) of } \\
\text { 30-degrees }\end{array}$ \\
\hline HRF & $\begin{array}{c}\text { High-Resolution Fundus } \\
\text { (HRF) Image } \\
\text { Database [12]. }\end{array}$ & $15 N / 15 G$ & $3504 \times 2336$ & \\
\hline ORIGA $A^{-l i g h t}$ & $\begin{array}{l}\text { ORIGA-light:An } \\
\text { Online Retinal Fundus } \\
\text { Image Database for } \\
\text { Glaucoma Analysis and } \\
\text { Research [13]. }\end{array}$ & 650 & $3072 \times 2048$ & $\begin{array}{l}\text { Singapore Malay Eye } \\
\text { Study. The authors } \\
\text { developed an in-house } \\
\text { retinal image grading } \\
\text { tool:ORIGAGT }\end{array}$ \\
\hline RIMONE & $\begin{array}{l}\text { RIM-ONE: An Open } \\
\text { Retinal Image Database } \\
\text { for Optic Nerve } \\
\text { Evaluation [14]. }\end{array}$ & $\begin{array}{l}118 \mathrm{~N} / 12 \mathrm{EG} \\
/ 14 \mathrm{MG} / 14 \mathrm{DG} \\
/ 11 \mathrm{OHT}\end{array}$ & & $\begin{array}{l}\text { The camera used to } \\
\text { capture these images is a } \\
\text { fundus camera Nidek } \\
\text { AFC-210 with a body of } \\
\text { a Canon EOS 5D Mark II } \\
\text { of } 21.1 \text { megapixels. }\end{array}$ \\
\hline ACRIMA & $\begin{array}{l}\text { CNNs for automatic } \\
\text { glaucoma assessment } \\
\text { using fundus images: an } \\
\text { extensive validation [15]. }\end{array}$ & $309 N / 396 G$ & & $\begin{array}{l}\text { They were captured } \\
\text { using the Topcon TRC } \\
\text { retinal camera and } \\
\text { IMAGEnet } \AA \text { capture } \\
\text { System. Images were } \\
\text { taken with a field of view } \\
\text { of } 35^{\circ} \text {. }\end{array}$ \\
\hline Tajimi & $\begin{array}{l}\text { The Tajimi study report } \\
\text { 2:prevalence of primary } \\
\text { angle closure and } \\
\text { secondary glaucoma in a } \\
\text { Japanese population [16]. }\end{array}$ & $\begin{array}{c}162(81 \\
\text { NFLDs }, 81 \mathrm{~N}) \\
261(130 \\
\text { NFLDs ,131 } \\
\mathrm{N})\end{array}$ & & $\begin{array}{c}\text { IMAGEnet digital fundus } \\
\text { camera system } \\
\text { (TRC-NW6S, Topcon, } \\
\text { Tokyo, } \\
\text { Japan)768×576 JPEG }\end{array}$ \\
\hline OIA-ODIR & $\begin{array}{c}\text { https://github.com/ } \\
\text { nkicsl/OIA }\end{array}$ & 10000 & & $\begin{array}{l}\text { They classify patient into } \\
\text { eight labels including } \\
\text { normal }(N) \text {, diabetes } \\
(D) \text {, glaucoma }(G), \\
\text { cataract }(C), A M D(A), \\
\text { hypertension }(H), \\
\text { myopia }(M) \text { and other } \\
\text { diseases/abnormalities } \\
(\mathrm{O}) \text { based on both eye } \\
\text { images and additionally } \\
\text { patient age. }\end{array}$ \\
\hline MESSIDOR & $\begin{array}{l}\text { Methods to evaluate } \\
\text { segmentation and } \\
\text { indexing techniques in } \\
\text { the field of retinal } \\
\text { ophthalmology [17]. }\end{array}$ & 1200 & $\begin{array}{l}1440 \times 960 \\
/ 2240 \times 1488 \\
/ 2304 \times 1536\end{array}$ & $\begin{array}{l}\text { The } 1200 \text { eye fundus } \\
\text { color numerical images of } \\
\text { the posterior pole of the } \\
\text { Messidor database were } \\
\text { acquired by } 3 \\
\text { ophthalmologic } \\
\text { departments using a } \\
\text { color video } 3 \text { CCD camera } \\
\text { mounted on a Topcon } \\
\text { TRC NW6 non-mydriatic } \\
\text { retinograph with a } 45 \\
\text { degree field of view. }\end{array}$ \\
\hline LAG & $\begin{array}{l}\text { A Large-Scale Database } \\
\text { and a CNN Model for } \\
\text { Attention-Based } \\
\text { Glaucoma Detection [18]. }\end{array}$ & $\begin{array}{l}11760(4878 \\
\text { positive } \\
\text { glaucoma } \\
\text { /6882 negative } \\
\text { glaucoma ) }\end{array}$ & & \\
\hline
\end{tabular}

${ }^{*} \mathrm{~N}$ stands for normal. 


\section{Background}

\section{Overview of Glaucoma Data Sets}

At present, there are many kinds of glaucoma public data sets, and many famous glaucoma data sets are shown in Table 1. These data sets have the following characteristics.

- The number of glaucoma medical image is small, which can not support the network with large FLOPs for training.

- Most medical image are fundus photos, which lack diversity and are not conducive to improving the accuracy of glaucoma diagnosis

- Most glaucoma data sets contain only two labels, namely normal and glaucoma. This has a disadvantage, that is, it can't go deep into glaucoma subtypes.

- Data sets are collected by different devices, which leads to a huge gap between images due to angle, light intensity, background and other reasons.

Because of the complexity of glaucoma, professional doctors need to make comprehensive diagnosis with the help of various medical images in the process of glaucoma diagnosis. If the neural network used in clinical diagnosis only uses one type of medical image, it will greatly reduce the accuracy of glaucoma diagnosis. Therefore, a multi-modal glaucoma medical image is urgently needed. At the same time, the labels of glaucoma data sets published at present are limited to the two-classification problem, which cannot solve the clinical problems of glaucoma subtypes that need more detailed labels, and a glaucoma data set with multi-classification labels is urgently needed. The data set we built perfectly solves the above two problems.

\section{Overview of glaucoma diagnostic models.}

\section{1) Retinal Vessel Segmentation}

At present, most of the RV segmentation tasks are carried out on the color fundus images [19-21]. At present, a device with high resolution has been introduced into glaucoma diagnosis, that is, OCTA. OCTA is a new non-invasive fundus imaging technique, which can identify retinal and choroidal blood flow information with high resolution and image retinal and choroidal microvascular circulation in vivo.Several RV segmentation methods in OCTA images have been reported [22,23].

\section{2) Single Modal Convolution Network for Glaucoma}

Deep learning has made significant achievements in image classification and semantic segmentation.It can fulfill the task of classification or segmentation end to end. Fu et al. [24]introduce a deep learning technique to gain additional imagerelevant information and screen glaucoma from the fundus image directly. Chen et al. [25] develop a deep learning (DL) architecture with convolutional neural network for automated glaucoma diagnosis. The proposed DL architecture contains six learned layers: four convolutional layers and two fully-connected layers. A segmentation method using Convolutional Neural Networks (CNN) has been implemented, in 2019, by Mamta Juneja [26]. The proposed system uses two neural networks working in conjunction to segment optic cup and disc.

Hovever,training a CNN from scratch is not an easy task. They require a huge amount of labelled data a requirement that is difficult to meet in the glaucoma assessment task and computational resources.In order to reduce the overfitting problem caused by the small amount of training samples, Hao Chen et al [27], proposed 
a transfer learning strategy, which transfers the knowledge in the low layers of a base CNN trained from a large database of natural images to their task-specific CNN.

In the study made by Abbas [28], he developed and implemented a system known as Glaucoma-Deep. This system consists of an unsupervised CNN architecture that automatically extracts features from the fundus images.

\section{3) Multimodal Methods for Glaucoma}

Multimodal machine learning is a new trend that builds models and relates information from multiple modalities. This is a dynamic multidisciplinary field that is increasingly important and has extraordinary potential [29]. Most of our known work is still focused on glaucoma single-modal network or heuristic learning. Up to now, no researchers proposed an effective multimodal diagnosis network for glaucoma. In order to fill the gap in this research, we carry out relevant research. A multimodal intelligent diagnosis network for glaucoma is proposed by us.

\section{Experimental Results}

To prove the excellence of the data set and the effectiveness of the multimodal network, we carried out three experiments. Experiment 1 mainly validates the validity of the data. Five fundus photo data sets and two OCT data sets are used to compare with our data sets. Experiment 2 mainly compares the indexes of multimodal neural networks under different backbone. Experiment 3 mainly compares the indexes of single-mode neural network and multi-mode neural network, thus verifying the excellence of multi-mode neural network. To evaluate the performance of the data set, The dataset is split into $70 \%$ training set , $10 \%$ valid set and $20 \%$ valid set with a stratified train-test split method to ensure the same proportion of each class. Each image and its corresponding metadata in the training set and test set are unique and irrelevant.All models in the experiments arefine-tuned for 300 epochs using the Adam optimization with a starting learning rate of 0.0001 . The batch size is equal to 32. MulTiNet is implemented based on Pytorch and Tensorflow. All the experiments were run on NVIDIA Tesla A100.

In order to solve the problems arising from the training model of medical image data sets, we introduce pre-training weights into the proposed model, and use glaucoma photos to train the last few full connection layers or classification layers.In transfer learning, it should be noted that the data preprocessing method must be the same as that of the pre-training model, otherwise the model will not converge.

The diagnosis of glaucoma is a multiclass classification problem. To evaluate the model comprehensively and compare the model objectively, we use multidimensional indicators, including accuracy (ACC), sensitivity (SEN), specificity (SPE), and area under the receiver operating characteristics (AUCs) of all diagnosis categories.

\section{Experiment1:Data Set Validity Experiment}

In this experiment, we choose EfficientNet as the backbone, which achieves stateof-the-art performance with fewer parameters compared with other models. And we compare the public data set (Drishti-GS, HRF, RIMONE, ACRIMA ...) with our data set. The results are shown in figure2 and table2.

In order to obtain the best performance of each model, we carried out several experiments varying the number of fine-tuned layers and the number of epochs. 


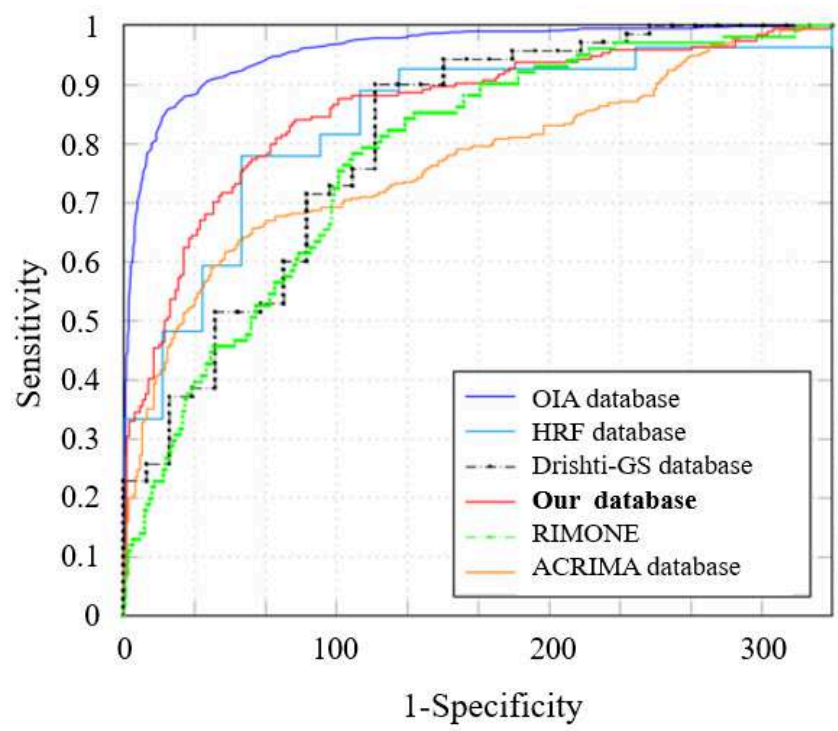

Figure 2 ROC curves for the EfficientNet backbone in different database.

Table 2 Training results under EfficinetNet

\begin{tabular}{cccccc}
\hline Database & images & ACC & SEN & AUC & AUC's 95\% confidence interval \\
\hline Drishti-GS & 101 & 0.816 & 0.813 & 0.817 & $55.48 \% \sim 92.55 \%$ \\
HRF & 30 & 0.828 & 0.833 & 0.825 & $50.00 \% \sim 100.00 \%$ \\
ORIGA-light & 650 & 0.800 & 0.793 & 0.786 & $65.48 \% \sim 87.54 \%$ \\
RIMONE & 455 & 0.856 & 0.859 & 0.862 & $74.33 \% \sim 89.36 \%$ \\
ACRIMA & 705 & 0.806 & 0.825 & 0.867 & $70.44 \% \sim 87.85 \%$ \\
OIA & 10000 & 0.937 & 0.943 & 0.957 & $92.26 \% \sim 98.55 \%$ \\
Ours & $\mathbf{3 1 7}$ & $\mathbf{0 . 8 7 8}$ & $\mathbf{0 . 8 5 6}$ & $\mathbf{0 . 8 6 6}$ & $\mathbf{8 0 . 2 2} \% \sim \mathbf{9 2 . 5 5 \%}$ \\
\hline
\end{tabular}

It can be seen from the table that training data sets with less images such as HRF under the network with larger FLOPs has lower accuracy and other indicators. Although the total number of our data sets is equivalent to that of RIMONE and other data sets, and the accuracy of the model is roughly the same, our data set contains 5 labels. Therefore, compared with the medical image data set with two classification labels, our data set still greatly improves the accuracy of glaucoma diagnosis, and can penetrate diagnostic keywords into glaucoma subtypes, which is not available in previous research work. In addition, using our data set for glaucoma diagnosis can not be limited to CDR features, but multi-feature extraction, which will enhance the richness and diversity of diagnosis. The network precision trained by our data is not as good as that trained by OIA, which is determined by the number of OIA data sets.

\section{Experiment2: Comparison the classification ability of different networks based on Transfer Learning.}

We compare the model trained by transfer learning method with the corresponding model not trained by transfer learning method to verify the effectiveness of introducing transfer learning.

In this paper, we use different convolution neural networks as Backbone, and use transfer learning method to carry out comparative experiments. The experimen- 


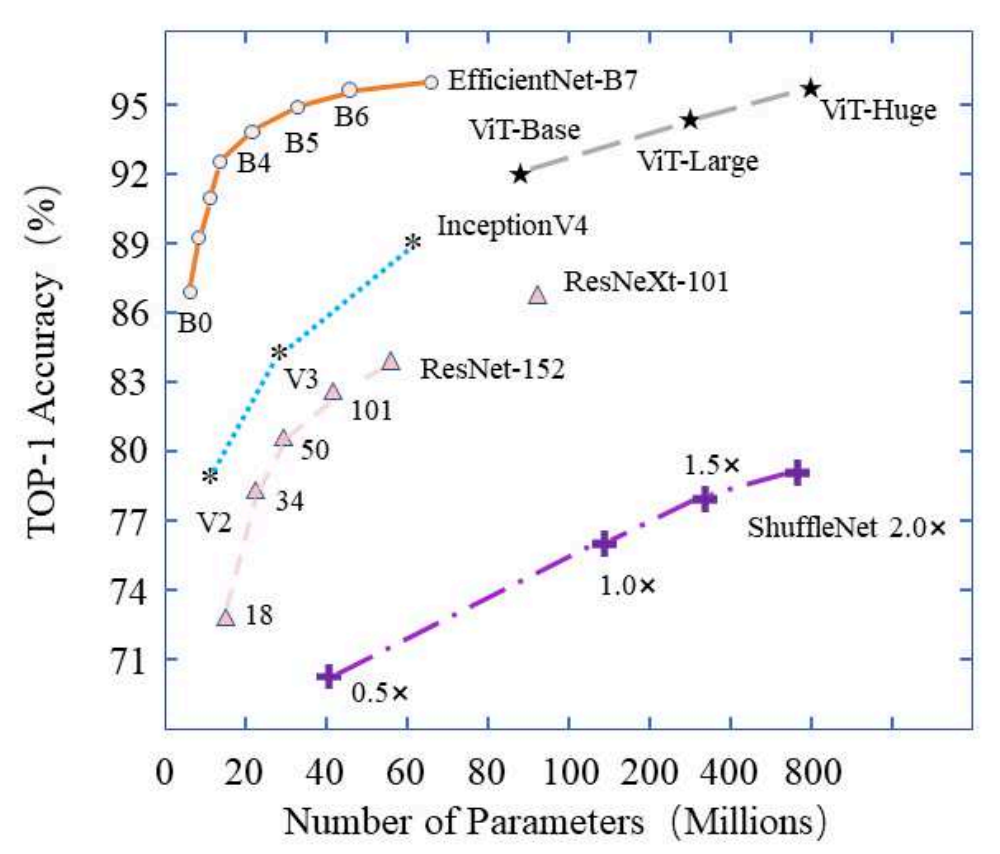

Figure 3 Model Size vs. Accuracy.

All models are trained on ImageNet using transfer learning method.

Table 3 Comparison between singlemodel network and multimodel network.

\begin{tabular}{ccccc}
\hline Networks & Pre-training weights & ACC & SEN & AUC \\
\hline \multirow{2}{*}{ ResNet50 } & Yes & 0.876 & 0.676 & 0.891 \\
& No & 0.693 & 0.421 & 0.706 \\
\hline \multirow{2}{*}{ InceptionV3 } & Yes & 0.891 & 0.765 & 0.894 \\
& No & 0.696 & 0.440 & 0.701 \\
\hline \multirow{2}{*}{ DenseNet121 } & Yes & 0.907 & 0.708 & 0.906 \\
& No & 0.678 & 0.453 & 0.692 \\
\hline \multirow{2}{*}{ EfficientNetB5 } & Yes & 0.933 & 0.724 & 0.876 \\
& No & 0.726 & 0.493 & 0.746 \\
\hline \multirow{2}{*}{ ViT } & Yes & 0.913 & 0.704 & 0.816 \\
& No & 0.715 & 0.506 & 0.727 \\
\hline \multirow{2}{*}{ ours } & Yes & $\mathbf{0 . 9 4 7}$ & $\mathbf{0 . 8 5 4}$ & $\mathbf{0 . 8 7 6}$ \\
& No & 0.733 & 0.556 & 0.747 \\
\hline & & & &
\end{tabular}

tal results are shown in the figure 3 and table3. EfficientNet has a good balance among receptive field $W$, network depth $D$ and resolution $R$, so it has achieved good results in glaucoma classification. Although Vision Transformer achieve good results in accuracy, more data will be used to achieve the same accuracy due to the large FLOPs which wastes a lot of computing resources. In the experiment, for InceptionV2, V3 and V4 networks, we found that using auxiliary classifier will greatly improve the accuracy of glaucoma classification. At the same time, ResNet can extract CDR feature well.

Experimental results show that our proposed model performs best among all models, with ACC of 0.947 , SEN of 0.854 and AUC of 0.876 . 


\section{Experiment 3: Comparison between singlemodel network and multimodel network.}

In order to validate the effectiveness of introducing multimodality, we compare the proposed model with single-modal modals. We evaluate three branches to measure the contribution of each branch.In the table 4, Branch1 contains only the text information of patients, such as gender, age, IOP, doctor's diagnosis, etc. Branch2 contains the characteristic information processed from medical images. Branch 3 contains original medical images. EfficientNet is used as Bcakbone in Branch 3.Multi-output refers to the output of a multimodal neural network.

Table 4 Comparison with Single-Modal Models.

\begin{tabular}{ccccc}
\hline Branch & ACC & SEN & AUC & AUC_SEN_80 \\
\hline Branch1 & 0.913 & 0.486 & 0.867 & 0.722 \\
Branch2 & 0.889 & 0.559 & 0.836 & 0.636 \\
Branch3 & 0.933 & 0.752 & 0.921 & 0.763 \\
\hline Muliti-output & 0.946 & 0.835 & 0.978 & 0.958 \\
\hline
\end{tabular}

From Table 4, the performance of the multimodal model is superior to any singlemodal ones.

\section{Discussion}

At present, in the process of medical diagnosis, the clarity of medical images often determines the diagnostic accuracy. The resolution of medical images usually exceeds 1000, which is far beyond the resolution of $224 \times 224$ of the usual image classification network. If the image is directly input into the network, the training time will be greatly increased. If the image is cropped, the pathological features will be lost, which will lead to the decline of diagnostic accuracy.How to increase the size of receptive field? This is critical for object detection in high-resolution images,especially for medical diagnosis. Therefore, how to solve such a problem will soon become a problem we will consider.

There are some problems in the training process. First, the network parameters can not reach the optimal. When HRF, a small amount of medical image data set, is used alone to input into the neural networks, the model can not fully learn the relevant characteristics of glaucoma. Because of the large number of network parameters and few data sets, the networks can not achieve better results. Second, the samples are unbalanced. For example, due to the imbalance between glaucoma samples and normal samples when we use OIA data set, the fitting effect in training set is better, but the accuracy in test set is very low, resulting in over-fitting problem. Therefore, we need to adjust the network according to the data characteristics.For example, for OIA data sets, we take down sampling, reduce other samples, and make them consistent with glaucoma data as much as possible.

\section{Conclusion}

In this article, we constructed a brand-new glaucoma data set with five labels and multiple medical images. As far as we know, this is the first multi-tagged glaucoma data set, and the first tag goes deep into the glaucoma subtype data set. At the same time, we construct a multimodal neural network. Three-branch structure is embedded in the network, and the text information and image information are fused 
at the end. The results of many experiments show that our multimodal glaucoma diagnosis model is superior to the convolution neural network proposed at present.

This data set and network will greatly improve the accuracy of glaucoma diagnosis, help alleviate the shortage of glaucoma professionals at present, and facilitate the diagnosis of suspected glaucoma patients as soon as possible. It is also helpful to discover new knowledge in the field of glaucoma diagnosis from multimodal information.

The future work will be divided into two parts: we will further enrich our data set, collect the time series data of intraocular pressure, transform the current glaucoma diagnosis problem into a prediction problem, and further enhance the early detection of glaucoma; We will further improve the accuracy of multimodal neural network and enhance the performance of the model.

\section{Material and methods}

\section{A.DaulSY:a new public database.}

Our data set involved 317 individuals, each of whom collected Heidelberg OCT, picture of retinal nerve fiber layer and fundus photos, and some individuals have UBM, SLO, OCTA and intraocular pressure data. Our data were reviewed by six professional glaucoma attending physicians, each with more than 10 years of clinical experience.First, the image is processed by four attending physicians, and the most interesting areas are marked, and the images are labeled. If the four physicians have the same label, the image will be used directly, otherwise it will need to be reviewed by two chief physicians with more than 20 years of clinical experience.Note that our database is constructed according to the tenets of the Declaration of Helsinki.

OCT, a non-contact and non-invasive imaging technology, has been widely employed to provide high-resolution images of optic nerve head (ONH) and retinal nerve fiber layer (RNFL) for structure assessment [30]. The effectiveness of intelligent diagnosis of glaucoma using Heidelberg OCT has been verified.

SLO provides a fine detailed vascular network which prepares enough vascular information to ensure that the quality of registration will be acceptable [31]. The OCTA is a new non-invasive imaging modality, which captures the blood vasculature network in various plexuses to indicate different layers of the retina. By using OCTA imaging modality,the ophthalmologist can easily examine avascular capillary,superficial capillary, and deep capillary plexuses in addition to choroid and choriocapillaris plexuses separately. The other advantage of OCTA is to allow ophthalmologists to measure foveal avascular zone (FAZ) and nonperfusion area bilaterally without obscuration by leakage of fluorescein dye [32].

Images in the dataset are grading into N, POAG, PACG, PACS, and PAC. Our dataset includes five labels, as shown in the figure 4.

\section{B.Preprocessing}

The purpose of preprocessing for image and metadata is to reduce the influence of noise and imbalanced classes in the data set, so as to improve the learning ability of the model for important features hidden in metadata and images. In deep learning (image field), data augmentation is a very common means to increase the number of training samples, especially for the small number of medical data.Data preprocessing is carried out for images and metadata.For example: 


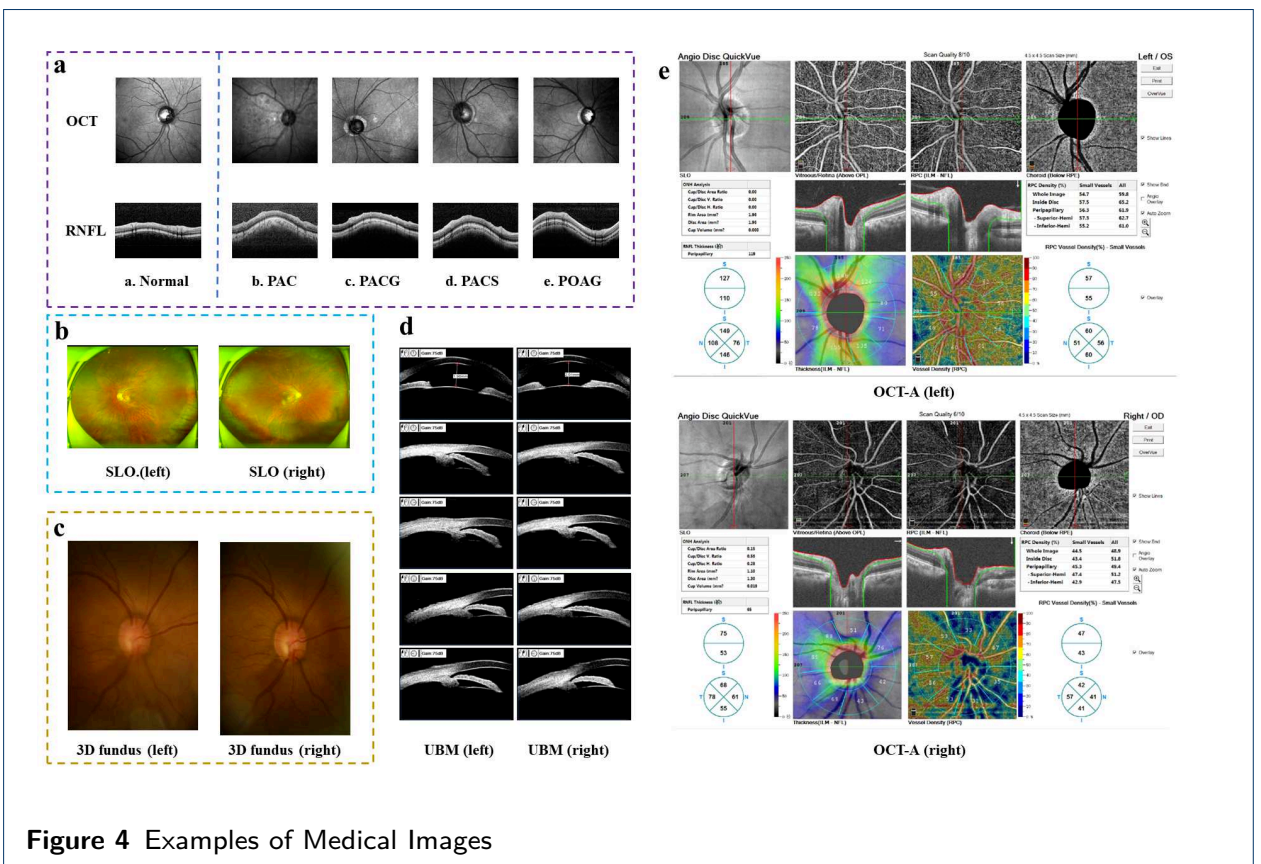

- Random horizontal flip.

- Random tone (H), saturation (S), lightness (V) adjustment.

- Random rotation, scaling, translation and miscutting.

- Mixup and Mosaic

The resolution of Heidelberg OCT and ocular fundus photos is as high as thousands. Although the accuracy of the model can be increased to some extent, it will greatly increase the training time of the model. Therefore, we choose to preprocess the images to adapt to the training of the model. The fundus images used for the fine-tuning process were automatically cropped around the optic disc using a bounding box of 1.5 times the optic disc radius. To do this cropping, we employed the method proposed in [33]. In their method, $\mathrm{Xu}$ et al. used a basic CNN to find the most probable pixels in the optic disc region. Then, they sort out those candidate pixels via using a threshold.

Cropping the images around the optic disc has a clinical reason, glaucoma disease affects mainly the optic disc and its surroundings. At the same time, we usually standardize the image in the process of image preprocessing, which can accelerate the convergence of the network.

\section{C.Feature Extraction}

When dividing the optic disc and the optic cup area, We use a popular deep learning model U-Net [34], a well-known U-shaped structure model based on FCN, which is composed of down-sampling and up-sampling structures similar to English capital letter $\mathrm{U}$, and use jumping connection at the same stage, thus ensuring that more high-level and low-level features are integrated into the final feature map.

\section{D.Fully Supervised Classification}

The flowchart of network is shown in Figure 5. This network consists of three paths (branches). The first branch deals with basic information of patients, such as age 


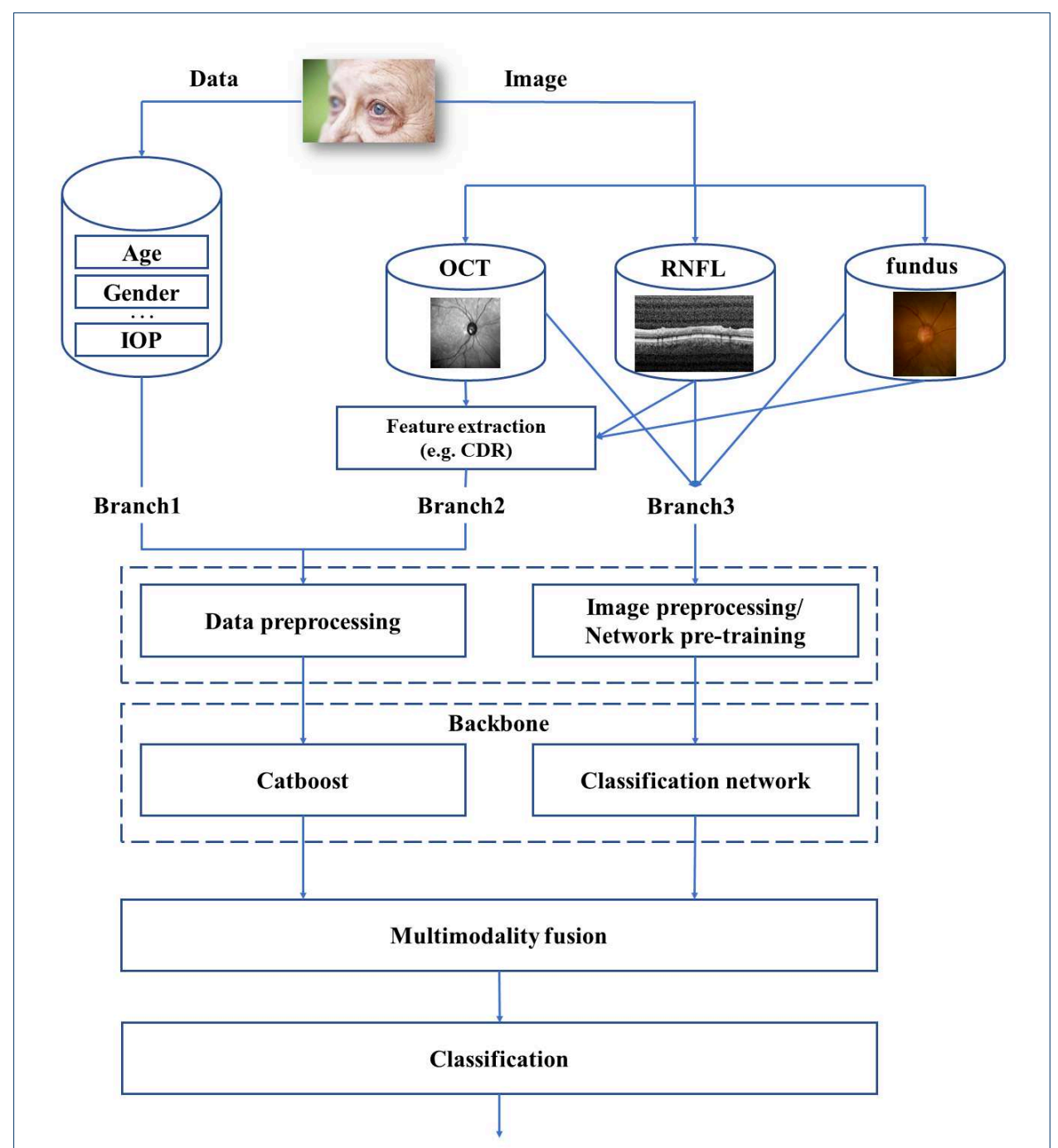

Figure 5 Flowchart of multimodal diagnosis of glaucoma

and gender, CDR and so on. The second branch introduces image features into metadata. It uses segmentation neural network to segment glaucoma optic disc and optic cup, and uses segmentation network to extract image features. The third branch focuses on glaucoma multimodal medical images, and uses advanced deep learning model sets as the backbone to capture global and local details.

Branch 1 and branch 2 are blended together as the metadata and processed with the Catboost classifier. Catboost is a popular ensemble boosting method that introduces an ordered target statistic strategy to deal with categorical features effectively. It replaces the category features ranked before the sample with the expectation of the feature value, and adds the priority and its weight. So, it can convert the category features into numerical features, effectively reducing the noise of low-frequency categorical feature and enhancing the robustness of the algorithm. 


\section{Resnet}

Resnet [35]was proposed by Microsoft Lab in 2015, and won the first place in classification task and target detection in ImageNet competition that year.

Highlights of the network:

- Ultra-deep network structure.

- The residual module is proposed.

- Abandon dropout and use BN to speed up training.

Simply stacking convolution layer and pooling layer may cause gradient disappearance or gradient explosion, which cannot increase the accuracy of the model. Resnet uses residual module and BN layer to standardize data, which can effectively solve the problem of gradient disappearance and gradient explosion.

Both Heidelberg OCT and fundus photos sampled the area around the optic disc. After many times of convolution, the feature extraction is roughly the same, so we fuse the two kinds of information in conv3 when we build the network. The optic nerve fiber layer thickness image is an expanded image of the first two optic nerve fiber layers, and the extracted feature is the optic nerve fiber layer thickness, so the image convolves five blocks separately. Then, the three are combined and entered into the average pool together. The network flowchart is shown in Figure 6.

Residual blocks can be expressed as:

$$
\left\{\begin{array}{l}
y_{l}=h\left(x_{l}\right)+F\left(x_{l}, W_{l}\right) \\
x_{l+1}=f\left(y_{l}\right)
\end{array}\right.
$$

Where $x_{l}$ and $x_{l+1}$ represent the input and output of the $l$-th residual cell, respectively. Note that each residual cell generally contains a multi-layer structure. $F$ is the residual function, representing the learned residual, $h\left(x_{l}\right)=x_{l}$ is the identity map, and $f$ is the ReLU activation function. Based on the above formula, we obtain the learning characteristics from shallow $l$ to deep $L$ as follows:

$$
x_{L}=x_{l}+\sum_{i=l}^{L-1} F\left(x_{i}, W_{i}\right)
$$

Using the chain rule, the gradient of the reverse process can be obtained:

$$
\frac{\partial \text { loss }}{\partial x_{l}}=\frac{\partial \text { loss }}{\partial x_{L}} \cdot \frac{\partial x_{L}}{\partial x_{l}}=\frac{\partial \text { loss }}{\partial x_{L}} \cdot\left(1+\frac{\partial}{\partial x_{L}} \sum_{i=l}^{L-1} F\left(x_{i}, W_{i}\right)\right)
$$

Resnet can adjust the network size by changing the number of blocks. This paper uses 18-layer network, 34-layer network, 50-layer network, 101-layer network and 152-layer network for comparative test, and uses the pre-training model given in Pytorch official document for Transfer learning.

At the same time, the ResNet block is simply modified to ResNeXt block, and the group is set to 32 for comparative experiment. We also changed BN layers in resnet network to GN layers to compare accuracy. 


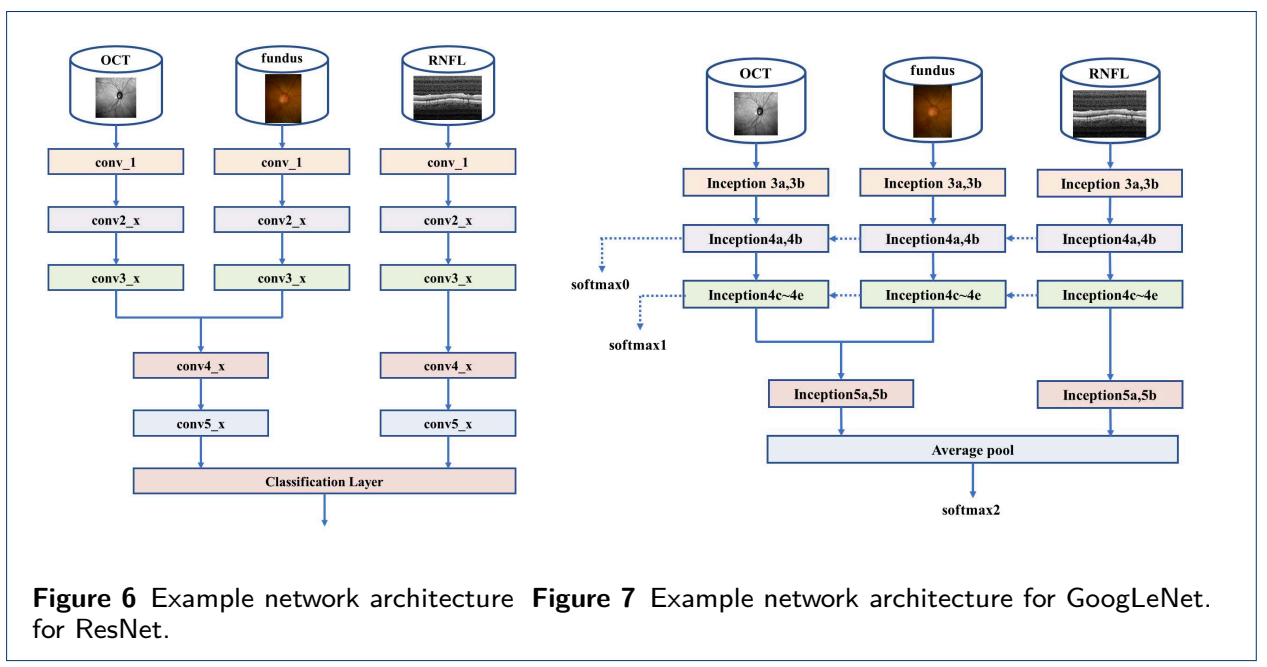

\section{GoogLeNet}

GoogLeNet [36] was proposed by Google team in 2014 and won the first place in Classification Task in ImageNet competition that year.

Inception uses convolution kernels of different sizes, which increases the adaptability of the network to different scales, and enhances the ability of feature expression (more convolution kernels, wider network and richer and more diverse information extracted), so that the network can freely choose better features and realize cross-channel interaction and information integration. At the same time, discarding the full connection layer and using the average pooling layer greatly reduces the model parameters, which is about one twelfth of Alexnet's parameters. Through $1 \times 1$ convolution kernel, dimension reduction and dimension increase are realized, and the purpose of parameter reduction is achieved. Among them, dimension reduction makes use of the characteristic that $1 \mathrm{x} 1$ convolution kernel can linearly combine features on different channels. Inception also uses intermediate nodes to assist classification, gives weight, uses the output of a certain intermediate layer to classify, plays the role of model fusion, and adds feedback gradient signal and extra regularization.

As shown in Figure 7,in the construction of multimodal neural network, we fuse the image information of Heidelberg OCT and fundus photos before Inception 5a. The thickness information of optic nerve fiber layer was fused before Average pool for comprehensive diagnosis of glaucoma.

\section{DenseNet}

One advantage of DenseNet is that the network is narrower and has fewer parameters.Each layer has direct access to the gradients from the loss function and the original input signal, leading to an implicit deep supervision [37].

After passing through $7 \times 7$ convolution layer and pooling layer, medical images go through Dense Block and Transition Layer three times. After that, Heidelberg OCT and fundus photo images are fused here, and the network is merged from three branches into two branches. After one Dense Block and Transition Layer, the 


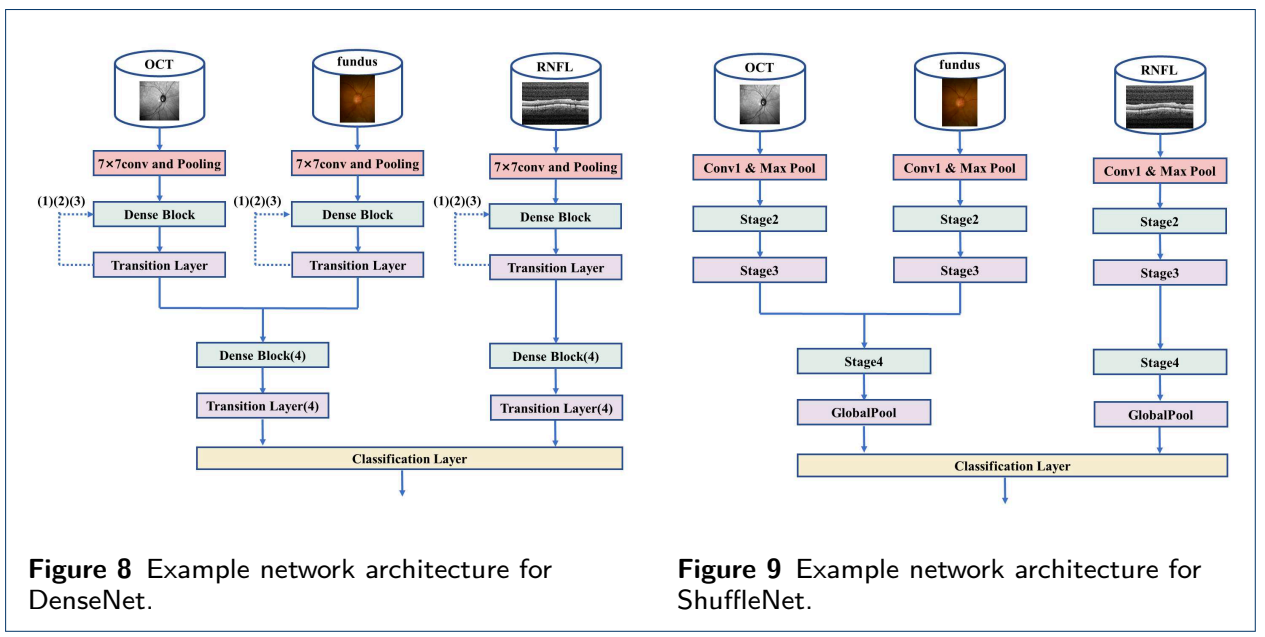

information of optic nerve fiber layer thickness is fused with others. The flow chart is shown in Figure 8.

$$
x_{l}=H_{l}\left(\left[x_{0}, x_{1}, \ldots x_{l-1}\right]\right)
$$

$\left[x_{0}, x_{1}, \ldots x_{l-1}\right]$ denotes concatenation of the 0 through $l-1$ layer feature map. $H_{l}$ includes BN, ReLU and the convolution of $3 * 3$.

\section{ShuffleNet}

One of ShuffleNet's great advantages is the use of group convolution. Group convolution reduces the parameters of the model effectively, which makes the training of the model faster [38,39].

Convolution Parameters: $H \times W \times C \times N$

GConv Parameters: $\left(H \times W \times \frac{C}{g} \times \frac{N}{g}\right) \times g$

where $g$ represents the number of Groups in GConv.If $\left\{\begin{array}{l}g=C \\ N=C\end{array}\right.$, the convolution is DW conv.

The network is mainly composed of a stack of ShuffleNet units.The first building in each stage is applied with stride $=2$. Other hyper-parameters within a stage stay the same,and for the next stage the output channels are doubled.If the number of input channels is relatively small, we do not apply group convolution on the first pointwise layer. The network flowchart is shown in Figure 9.

\section{EfficientNet}

There are many problems in EffcientV1 network [40], for example, when the size of the training image is very large, the training speed is very slow, and when using Depthwise solutions in the shallow layer of the network, the speed will be very slow, and it is suboptimal to enlarge each stage equally. Therefore, in this article, I use EfficientV2 network [41] to establish a glaucoma classification model.

Highlights of the network: 


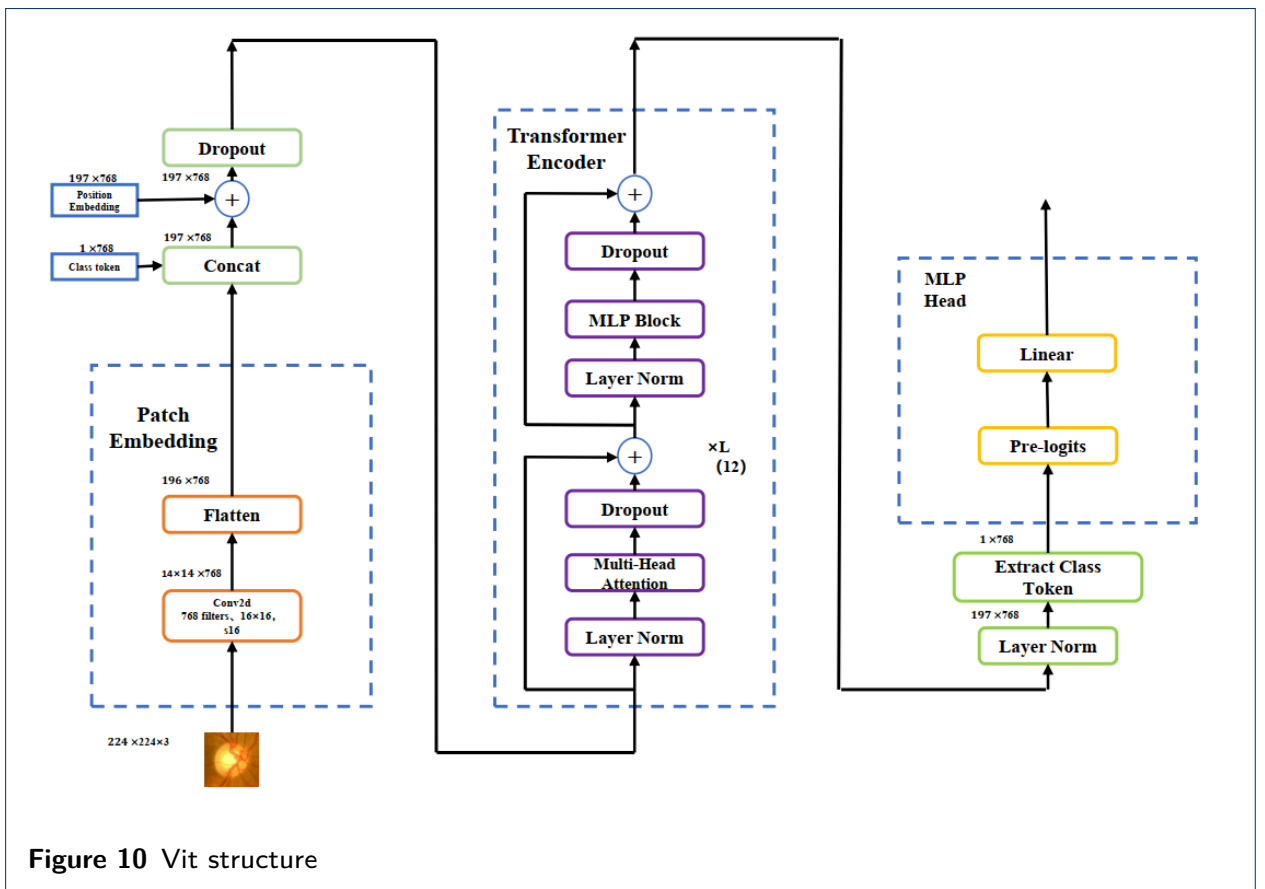

- Introducing Fused-MBConv block.

- Introduce progressive learning strategy (training is faster).

- Prefer to use smaller kernel-size $(3 \times 3)$

The author of the original EfficientV2 paper did not replace all MBConv with Fused-MBConv, but only replaced the MBConv module of 1 stage, 2 stage and 3 stage.

Efficient network not only has the accuracy reached the current SOTA level, but also has a faster training speed, which is increased by 11 times; The number of parameters is less, which is reduced by $\frac{1}{6.8}$. This network uses an improved progressive learning method, which dynamically adjusts the regularization method according to the size of the training image.

When expansion ratio is equal to 1, there is no expansion conv. A shortcut connection is only available when stride $=1$ and the input and output Channels are equal. Also note that there is a Dropout layer when there is a shortcut connection, and the Dropout layer here is Stochastic Depth, which randomly discards the main branch of the whole block.

\section{Vision Transformer}

Simply put, this model [42] consists of three blocks:

- Linear Projection of Flattened Patches(Embedding)

- Transformer Encoder

- MLP Head

As shown in the figure10,in order to obtain the token sequence of standard input, we use a convolution layer of 768 filters of kernel size $16 \times 16$ with stride 16 . Then use Flatten to flatten the $\mathrm{H}$ and $\mathrm{W}$ dimensions. Finally, add [class] token and Position Embedding. Then the matrix of embedding output is input into the Transformer Encoder.Transformer Encoder is essentially stacking the Encoder Block L times. As 
shown in the figure, it is first input to LN layer (Why not use direct BN? Because in time series networks such as RNN, the length of time series is not a fixed value, that is, the network depth is not necessarily the same, for example, the length of each sentence is not necessarily the same, so it is difficult to use BN), and then the multi-head attention mechanism is used to process the data, followed by the dropout layer.

$$
\operatorname{Attention}(Q, K, V)=\operatorname{softmax}\left(\frac{Q K^{T}}{\sqrt{d_{k}}}\right) V
$$

\section{E.Focal loss}

Imbalanced data are common in the medical field, which makes the classifier focus more on the major classes but neglect the minor classes. It results in a low sensitivity to the minor classes and a low specificity to the major classes, which can be addressed to a certain degree by revising the loss function.

The categorical cross-entropy loss is a popular loss function in multiclass classification learning. It assigns the same weight to each class, which leads to little attention to the minor classes and results in a low sensitivity for underrepresented classes. To overcome the effect of imbalanced data, we introduces focal loss as the loss function. Focal loss is a variant of the categorical cross-entropy loss, which has been proposed for handling imbalance data.

$$
\text { Focal }_{L} \text { oss }=-\sum_{i=0}^{N} \sum_{c=0}^{C} \alpha_{i, c} y_{i, c}\left(1-p_{i, c}\right)^{\gamma} \log p_{i, c}
$$

\section{Appendix}

\section{Acknowledgements}

Here, I would like to thank many attending physicians in the Fourth People's Hospital of Shenyang, China. They have made great contributions to the construction of this data set. Thanks to Professor Jiao Wang of Northeastern University for his guidance to this paper. Finally, thank myself for my hard work.

Funding

This work is supported by the Eye Disease Fund jointly applied by the Fourth People's Hospital of Shenyang, China and the Institute of Deep Learning and Advanced Intelligent Decision-making of Northeastern University of China.

Abbreviations

The authors declare that they have no competing interests.

Availability of data and materials

The data that support the findings of this study are available from Shenyang Fourth People's Hospital but restrictions apply to the availability of these data, which were used under license for the current study, and so are not publicly available. Data are however available from the authors upon reasonable request and with permission of Shenyang Fourth People's Hospital.

Ethics approval and consent to participate

Our database is constructed according to the tenets of the Declaration of Helsinki.

Competing interests

The authors declare that they have no competing interests.

Consent for publication

The medical images of all cases were obtained with the consent of the patients themselves, and we signed a consent form and a confidentiality agreement with them.

Authors' contributions

$\mathrm{Li}$ wrote the pape.Han,Zhao provided both technical and scientific writing support to this manuscript. All authors read and approved the final manuscript. 


\section{Author details}

${ }^{1}$ Department of Information Science and Engineering, Northeastern University, Shen Yang, China. ${ }^{2}$ Department of Information Science and Engineering, Northeastern University, Shen Yang, China. ${ }^{3}$ Department of Information Science and Engineering, Northeastern University, Shen Yang, China. ${ }^{4}$ Department of Information Science and Engineering, Northeastern University, Shen Yang, China. ${ }^{5}$ Department of Information Science and Engineering, Northeastern University, Shen Yang, China.

References

1. Kingman, Sharon: Glaucoma is second leading cause of blindness globally. Bulletin of the World Health Organization (2004)

2. Quigley, A, H.: The number of people with glaucoma worldwide in 2010 and 2020. British Journal of Ophthalmology 90(3), 262-267 (2006)

3. Meng, Z., Zhao, Z., Li, B., Su, F., Guo, L.: A cervical histopathology dataset for computer aided diagnosis of precancerous lesions. IEEE Transactions on Medical Imaging 40(6), 1531-1541 (2021) doi:10.1109/TMI.2021.3059699

4. Wang, S., Yin, Y., Wang, D., Wang, Y., Jin, Y.: Interpretability-based multimodal convolutional neural networks for skin lesion diagnosis. IEEE Transactions on Cybernetics, 1-15 (2021). doi:10.1109/TCYB.2021.3069920

5. Wang, X., Chen, H., Gan, C., Lin, H., Dou, Q., Tsougenis, E., Huang, Q., Cai, M., Heng, P.-A.: Weakly supervised deep learning for whole slide lung cancer image analysis. IEEE Transactions on Cybernetics 50(9), 3950-3962 (2020). doi:10.1109/TCYB.2019.2935141

6. Ovreiu, S., Paraschiv, E.-A., Ovreiu, E.: Deep learning amp; digital fundus images: Glaucoma detection using densenet. In: 2021 13th International Conference on Electronics, Computers and Artificial Intelligence (ECAI), pp. 1-4 (2021). doi:10.1109/ECAI52376.2021.9515188

7. Gisler, C., Ridi, A., Fauquex, M., Genoud, D., Hennebert, J.: Towards glaucoma detection using intraocular pressure monitoring. In: 2014 6th International Conference of Soft Computing and Pattern Recognition (SoCPaR), pp. 255-260 (2014). doi:10.1109/SOCPAR.2014.7008015

8. Liao, W., Zou, B., Zhao, R., Chen, Y., He, Z., Zhou, M.: Clinical interpretable deep learning model for glaucoma diagnosis. IEEE Journal of Biomedical and Health Informatics 24(5), 1405-1412 (2020) doi:10.1109/JBHI.2019.2949075

9. Hu, Z., Niemeijer, M., Abramoff, M.D., Garvin, M.K.: Multimodal retinal vessel segmentation from spectral-domain optical coherence tomography and fundus photography. IEEE Transactions on Medical Imaging 31(10), 1900-1911 (2012). doi:10.1109/TMI.2012.2206822

10. Kafieh, R., Rabbani, H., Hajizadeh, F., Ommani, M.: An accurate multimodal 3-d vessel segmentation method based on brightness variations on oct layers and curvelet domain fundus image analysis. IEEE Transactions on Biomedical Engineering 60(10), 2815-2823 (2013). doi:10.1109/TBME.2013.2263844

11. Sivaswamy, J., Krishnadas, S.R., Datt Joshi, G., Jain, M., Syed Tabish, A.U.: Drishti-gs: Retinal image dataset for optic nerve head(onh) segmentation. In: 2014 IEEE 11th International Symposium on Biomedical Imaging (ISBI), pp. 53-56 (2014). doi:10.1109/ISBI.2014.6867807

12. Budai, A., Bock, R., Maier, A., Hornegger, J., Michelson, G.: Robust vessel segmentation in fundus images. International Journal of Biomedical Imaging 2013(6), 154860 (2013). doi:10.1155/2013/154860

13. Zhang, Z., Yin, F.S., Liu, J., Wong, W.K., Tan, N.M., Lee, B.H., Cheng, J., Wong, T.Y.: Origa $<$ sup $>$-light $</$ sup $>$ : An online retinal fundus image database for glaucoma analysis and research. In: 2010 Annual International Conference of the IEEE Engineering in Medicine and Biology, pp. 3065-3068 (2010). doi:10.1109/IEMBS.2010.5626137

14. Fumero, F., Alayon, S., Sanchez, J.L., Sigut, J., Gonzalez-Hernandez, M.: Rim-one: An open retinal image database for optic nerve evaluation. In: 2011 24th International Symposium on Computer-Based Medical Systems (CBMS), pp. 1-6 (2011). doi:10.1109/CBMS.2011.5999143

15. Diaz-Pinto, A., Morales, S., Naranjo A, Köhler, T., Mossi, J.M., Navea, A.: Cnns for automatic glaucoma assessment using fundus images: An extensive validation. Biomedical Engineering Online 18(1) (2019)

16. Yamaomoto, T.: The tajimi study report 2: prevalence of primary angle closure and secondary glaucoma in a japanese population. Ophthalmology 112 (2005)

17. Decencière, E., Zhang, X., Cazuguel, G., Lay, B., Cochener, B., Trone, C., Gain, P., Ordonez, R., Massin, P. Erginay, A., Charton, B., Klein, J.-C.: Feedback on a publicly distributed database: the messidor database. Image Analysis \& Stereology 33(3), 231-234 (2014). doi:10.5566/ias.1155

18. Li, L., Xu, M., Liu, H., Li, Y., Wang, X., Jiang, L., Wang, Z., Fan, X., Wang, N.: A large-scale database and a cnn model for attention-based glaucoma detection. IEEE Transactions on Medical Imaging 39(2), 413-424 (2020). doi:10.1109/TMI.2019.2927226

19. Sheng, B., Li, P., Mo, S., Li, H., Hou, X., Wu, Q., Qin, J., Fang, R., Feng, D.D.: Retinal vessel segmentation using minimum spanning superpixel tree detector. IEEE Transactions on Cybernetics 49(7), 2707-2719 (2019) doi:10.1109/TCYB.2018.2833963

20. Roychowdhury, S., Koozekanani, D.D., Parhi, K.K.: Iterative vessel segmentation of fundus images. IEEE Transactions on Biomedical Engineering 62(7), 1738-1749 (2015). doi:10.1109/TBME.2015.2403295

21. Al-Diri, B., Hunter, A., Steel, D.: An active contour model for segmenting and measuring retinal vessels. IEEE Transactions on Medical Imaging 28(9), 1488-1497 (2009). doi:10.1109/TMI.2009.2017941

22. Eladawi, N., Elmogy, M., Helmy, O., Aboelfetouh, A., Riad, A., Sandhu, H., Schaal, S., El-Baz, A.: Automatic blood vessels segmentation based on different retinal maps from octa scans. Computers in Biology and Medicine 89, 150-161 (2017). doi:10.1016/j.compbiomed.2017.08.008

23. Li, M., Chen, Y., Ji, Z., Xie, K., Yuan, S., Chen, Q., Li, S.: Image projection network: 3d to 2d image segmentation in octa images. IEEE Transactions on Medical Imaging 39(11), 3343-3354 (2020). doi:10.1109/TMI.2020.2992244

24. Fu, H., Cheng, J., Xu, Y., Zhang, C., Wong, D.W.K., Liu, J., Cao, X.: Disc-aware ensemble network for glaucoma screening from fundus image. IEEE Transactions on Medical Imaging 37(11), 2493-2501 (2018). 
doi:10.1109/TMI.2018.2837012

25. Chen, X., Xu, Y., Kee Wong, D.W., Wong, T.Y., Liu, J.: Glaucoma detection based on deep convolutional neural network. In: 2015 37th Annual International Conference of the IEEE Engineering in Medicine and Biology Society (EMBC), pp. 715-718 (2015). doi:10.1109/EMBC.2015.7318462

26. Juneja, M., Singh, S., Agarwal, N.e.a.: Automated detection of glaucoma using deep learning convolution network (g-net). Multimed Tools Appl 79, 15531-15553 (2020). doi:10.1007/s11042-019-7460-4

27. Chen, H., Ni, D., Qin, J., Li, S., Yang, X., Wang, T., Heng, P.A.: Standard plane localization in fetal ultrasound via domain transferred deep neural networks. IEEE Journal of Biomedical and Health Informatics 19(5), 1627-1636 (2015). doi:10.1109/JBHI.2015.2425041

28. Abbas, Q.: Glaucoma-deep: Detection of glaucoma eye disease on retinal fundus images using deep learning. International Journal of Advanced Computer Science and Applications 8(6) (2017) doi:10.14569/IJACSA.2017.080606

29. Baltrušaitis, T., Ahuja, C., Morency, L.-P.: Multimodal machine learning: A survey and taxonomy. IEEE Transactions on Pattern Analysis and Machine Intelligence 41(2), 423-443 (2019). doi:10.1109/TPAMI.2018.2798607

30. Song, D., Fu, B., Li, F., Xiong, J., He, J., Zhang, X., Qiao, Y.: Deep relation transformer for diagnosing glaucoma with optical coherence tomography and visual field function. IEEE Transactions on Medical Imaging 40(9), 2392-2402 (2021). doi:10.1109/TMI.2021.3077484

31. Ghasemi Kamasi, Z., Mokhtari, M., Rabbani, H.: Non-rigid registration of fluorescein angiography and optical coherence tomography via scanning laser ophthalmoscope imaging. In: 2017 39th Annual International Conference of the IEEE Engineering in Medicine and Biology Society (EMBC), pp. 4415-4418 (2017). doi:10.1109/EMBC.2017.8037835

32. Eladawi, N., Elmogy, M., Fraiwan, L., Ghazal, M., Pichi, F., Aboelfetouh, A., Riad, A., Keynton, R., Schaal, S. El-Baz, A.: An octa based diagnosis system based on a comprehensive local features analysis for early diabetic retinopathy detection. In: 2018 IEEE International Conference on Imaging Systems and Techniques (IST), pp. 1-6 (2018). doi:10.1109/IST.2018.8577169

33. Xu P, Wan, C., Cheng, J., Niu, D., Liu, J.: Optic disc detection via deep learning in fundus images. Fetal, infant and ophthalmic medical image analysis., 134-141 (2017)

34. Ronneberger, O., Fischer, P., Brox, T.: U-net: Convolutional networks for biomedical image segmentation. In Navab, N., Hornegger, J., Wells, W.M., Frangi, A.F. (eds.) Medical Image Computing and Computer-Assisted Intervention - MICCAI 2015, pp. 234-241. Springer, Cham (2015)

35. He, K., Zhang, X., Ren, S., Sun, J.: Deep residual learning for image recognition. In: 2016 IEEE Conference on Computer Vision and Pattern Recognition (CVPR), pp. 770-778 (2016). doi:10.1109/CVPR.2016.90

36. Szegedy, C., Liu, W., Jia, Y., Sermanet, P., Reed, S.E., Anguelov, D., Erhan, D., Vanhoucke, V., Rabinovich, A.: Going deeper with convolutions. CoRR abs/1409.4842 (2014). 1409.4842

37. Huang, G., Liu, Z., Van Der Maaten, L., Weinberger, K.Q.: Densely connected convolutional networks. In: 2017 IEEE Conference on Computer Vision and Pattern Recognition (CVPR), pp. 2261-2269 (2017). doi:10.1109/CVPR.2017.243

38. Zhang, X., Zhou, X., Lin, M., Sun, J.: Shufflenet: An extremely efficient convolutional neural network for mobile devices. CoRR abs/1707.01083 (2017). 1707.01083

39. Ma, N., Zhang, X., Zheng, H., Sun, J.: Shufflenet V2: practical guidelines for efficient CNN architecture design CoRR abs/1807.11164 (2018). 1807.11164

40. Tan, M., Le, Q.V.: Efficientnet: Rethinking model scaling for convolutional neural networks. CoRR abs/1905.11946 (2019). 1905.11946

41. Tan, M., Le, Q.V.: Efficientnetv2: Smaller models and faster training. CoRR abs/2104.00298 (2021) 2104.00298

42. Dosovitskiy, A., Beyer, L., Kolesnikov, A., Weissenborn, D., Zhai, X., Unterthiner, T., Dehghani, M., Minderer, M., Heigold, G., Gelly, S., Uszkoreit, J., Houlsby, N.: An image is worth $16 \times 16$ words: Transformers for image recognition at scale. CoRR abs/2010.11929 (2020). 2010.11929 